\title{
Lymphomatoid granulomatosis presenting as tinnitus in a patient with acquired immunodeficiency syndrome
}

\author{
Renato Quispe, J Martin Rodriguez, David McCollum
}

\section{ABSTRACT}

Introduction: Puzzling neurologic symptoms in an immunosuppressed patient presents the clinician with a broad and challenging differential diagnosis. Multiple neurological complications in human immunodeficiency virus/acquired immunodeficiency syndrome (HIV/AIDS)-infected individuals have been reported, the most common being cognitive dysfunction, infections, and malignancies. In patients with advanced immunosuppression any neurological symptom should be taken seriously. We report a case of Epstein-Barr virus (EBV) related lymphomatoid granulomatosis presenting in a patient with AIDS initially as tinnitus. Case Report: A 42-year-old female was presented to her physician complaining of tinnitus. Unbeknownst to her physician, she had been diagnosed with human immunodeficiency virus (HIV) 14 years prior. Her tinnitus led her to undergo a workup in outpatient clinic. However, her neurological symptoms gradually progressed to include hearing loss and diplopia. She was admitted to another hospital and underwent an

Renato Quispe ${ }^{1}$, J Martin Rodriguez ${ }^{2}$, David McCollum ${ }^{3}$ Affiliations: ${ }^{1} \mathrm{MD}$, Clinical Research Associate, Instituto de Medicina Tropical "Alexander von Humboldt", Universidad Peruana Cayetano Heredia, Lima, PERÚ; ${ }^{2} \mathrm{MD}$, Assistant Professor of Medicine, Division of Infectious Diseases, University of Alabama at Birmingham, Birmingham, AL; ${ }^{3} \mathrm{MD}$, Assistant Professor of Medicine, Division of General Internal Medicine, University of Alabama at Birmingham, Birmingham, AL.

Corresponding Author: Renato Quispe, MD, Instituto de Medicina Tropical "Alexander von Humboldt", Universidad Peruana Cayetano Heredia, 430 Honorio Delgado Ave Ingenieria, San Martin de Porres, Lima. PERU. Lima 31; Ph: (511)987760463; Email: renato.quispe@upch.pe

Received: 15 October 2013

Accepted: 14 November 2013

Published: 01 April 2014 extensive array of testing. Laboratory testing indicated that her HIV had progressed to AIDS. Neurological imaging revealed a brainstem lesion. She was transferred to our hospital when the brainstem lesion was deemed inaccessible by neurosurgery. Several small pulmonary nodules had been noted on computed tomography scan of her chest. Surgical biopsy with pathology and flow cytometry of a lung nodule showed EBV-related lymphomatoid granulomatosis. Soon after, a unifying diagnosis between her lung nodules and a central nervous system disease was made as flow cytometry of her cerebrospinal fluid showed an identical aberrant B cell population consistent with lymphomatoid granulomatosis with transformation to diffuse large B cell lymphoma. Unfortunately, patient's brainstem lesions progressed and patient died after a few weeks in the hospital. Conclusion: Epstein-Barr virus related lymphomatoid granulomatosis is a rare disease that can be the cause of unexplained neurological symptoms in immunocompromised individuals. We report a challenging case in which the diagnosis was only considered after lung biopsy of relatively asymptomatic pulmonary nodules.

Keywords: Lymphomatoid granulomatosis, Epstein-Barr virus (EBV), Human immunodeficiency virus, Tinnitus

\section{How to cite this article}

Quispe R, Rodriguez JM, McCollum D. Lymphomatoid granulomatosis presenting as tinnitus in a patient with acquired immunodeficiency syndrome. International Journal of Case Reports and Images 2014;5(4):264-269.

doi: 10.5348/ijcri-201454-CR-10365 


\section{INTRODUCTION}

Human immunodeficiency virus (HIV) remains one of the world's most significant public health challenges, particularly in low and middle-income countries. According to the World Health Organization (WHO), 34 million people are living with HIV worldwide, and an estimated 2.5 million people were newly infected with the virus in 2011 [1]. Patients may present with a wide range of complications with diverse and puzzling symptoms, often involving multiple organ systems. While the epidemiology of neurological complications has changed considerably since the introduction of highly active antiretroviral therapy (HAART), a wide spectrum of neurological complications in HIV-infection individuals continues to be seen. Their development occurs due to a variety of mechanisms including loss of cell-mediated immunity, aberrant activation of the immune system, and HIV infection itself [2]. The vast majority are represented by opportunistic infections, mainly cryptococcal meningitis, cerebral toxoplasmosis, progressive multifocal leukoencephalopathy (PML), tuberculous meningitis and cytomegalovirus (CMV) encephalitis. Cognitive disorders are common in HIVinfected individuals, ranging from asymptomatic neurocognitive disorders to full blown HIV-dementia. Although less frequent, HIV-related malignant diseases (i.e., primary CNS lymphoma) should also be considered [3]. Peripheral neuropathies, such as HIV myelopathy and polyneuropathy, continue to be relatively common and significantly impact on quality of life [4]. When dealing with an HIV-infected patient presenting with neurological symptoms, comprehensive testing often needs to be performed with multiple etiologies sought and excluded. In this case, we present a previously untreated HIV-infected patient, who presented with progressive neurological symptoms. Eventually her symptoms were determined to be caused by Epstein-Barr virus (EBV)related lymphomatoid granulomatosis (LYG), a rare lymphoproliferative disorder that can transform into diffuse large B cell lymphoma [5].

\section{CASE REPORT}

A 42-year-old female with untreated HIV infection presented with a progressive neurological illness. For the previous two months, she had been noticing a feeling of fullness in her right ear and tinnitus. She was seen by an ENT physician who prescribed oral antibiotics and meclizine. Her symptoms progressed to involve her left ear, followed by bilateral hearing loss and diplopia. Review of systems was positive only for a dry cough for two months. She denied fever, shortness of breath or weight loss. She was diagnosed with HIV 14 years prior but never sought medical care. Otherwise she had no medical problems and took no medications.
The patient was admitted to an outside hospital, and was found to have bilateral ophthalmoplegia, nystagmus, and right-sided facial palsy. Initial laboratory studies included an unremarkable metabolic panel and mild leukopenia. Her CD4 count was 24 cells $/ \mathrm{mm}^{3}$ and HIV viral load was 33,000 copies/mL. Magnetic resonance imaging (MRI) scan of brain showed a single enhancing process of the right pons along with ependymal enhancement of the lateral ventricles. Cerebrospinal fluid (CSF) showed normal opening pressure, 84 white blood cells $/ \mathrm{mm}^{3}$ (91\% lymphocytes), glucose of $10 \mathrm{mg} / \mathrm{dL}$, and protein of $209 \mathrm{mg} / \mathrm{dL}$. Differential diagnosis at this time was extensive with an infectious etiology considered most likely, but malignancy also a serious consideration. Some of the most likely considerations included tuberculosis, CMV disease, Cryptococcus, toxoplasma, lymphoma, and PML. Negative CSF studies included gram stain/ culture, AFB smear/culture, cryptococcal antigen, VDRL, TB PCR, JC virus PCR, cytology and flow cytometry. The CSF studies were positive for CMV DNA (9,242 copies/ $\mathrm{mL})$ and EBV DNA (14,900 copies/mL). Negative studies of the serum and urine included bacterial and fungal cultures, RPR, PPD, QuantiFERON@-TB Gold, urinary Histoplasma antigen, toxoplasma antibody, and Aspergillus galactomannan. A bone marrow biopsy was unremarkable. Computed tomography (CT) scan of chest revealed a 2-cm left lower lung nodule and 12 smaller nodules scattered throughout the lungs. On bronchoscopy, bronchioalveolar lavage and biopsy revealed Pneumocystis jiroveci organisms and chronic inflammation without dysplasia; stains and cultures were otherwise negative. She received trimethoprim/ sulfamethoxazole, steroids, and empiric antituberculous therapy. Nevertheless, during the three weeks she spent at the outside hospital, her illness progressed to complete deafness, severe vertigo, unsteady gait, right-sided leg weakness and slurring of speech. She was transferred to our hospital.

At our institution the patient was continued on Pneumocystis and TB therapy with addition of ganciclovir given the positive CMV CSF PCR. Repeat brain MRI scan showed impressive progression after 11 days with enhancing lesions now throughout her brainstem, cerebellum, right inferolateral putamen, right thalamus, bilateral periventricular region and mild hydrocephalus (Figure 1). The CT scan of the chest again showed nodules, some with mild halo effect and cavitation (Figure 2).

The patient made no clinical improvement. At this point, the multiple negative laboratory tests for infectious etiologies, along with the lack of response to empiric tuberculosis and CMV treatment had shifted our differential diagnosis in favor of a CNS malignancy, particularly an aggressive lymphoma. Neurosurgery consult recommended against brain biopsy given the high-risk location of the lesions. Antiretroviral therapy for HIV was considered, but initially deferred due to concern for potential catastrophic Immune Reconstitution Inflammatory Syndrome (IRIS) phenomenon should the 


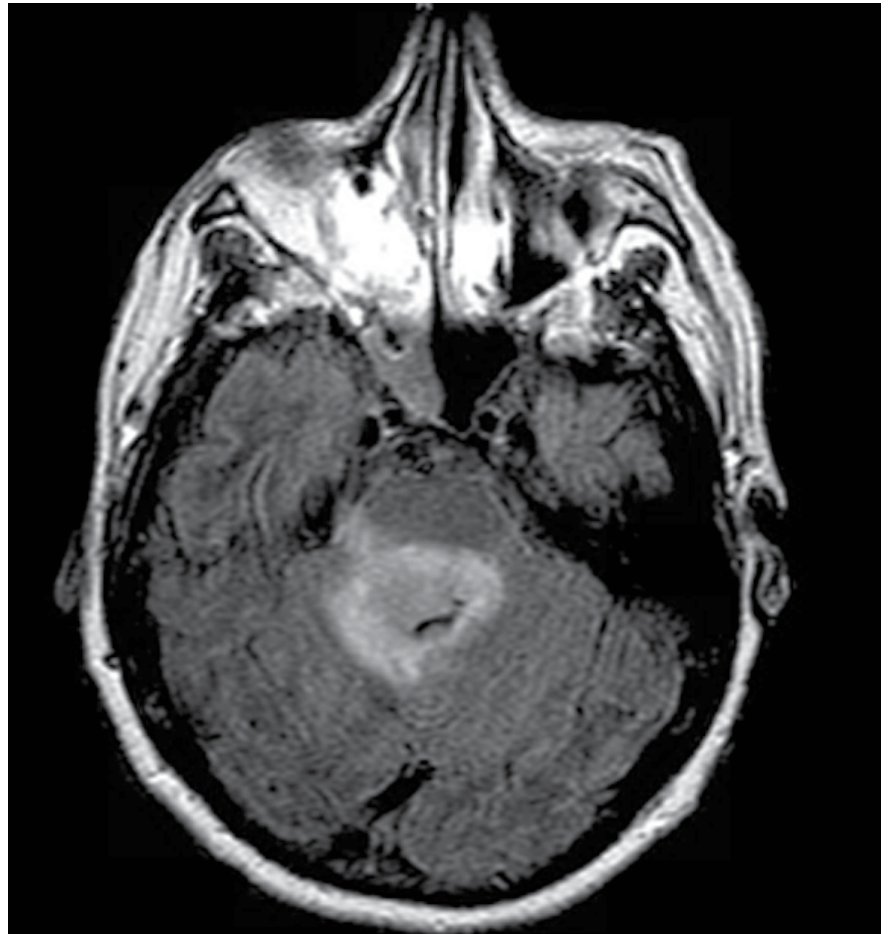

Figure 1: Magnetic resonance imaging of brain. T2/Flair imaging showing hyperintense process on in the brainstem with extension to cerebellum. Additionally, there were lesions present in the right thalamus and periventricular/ subependymal areas.

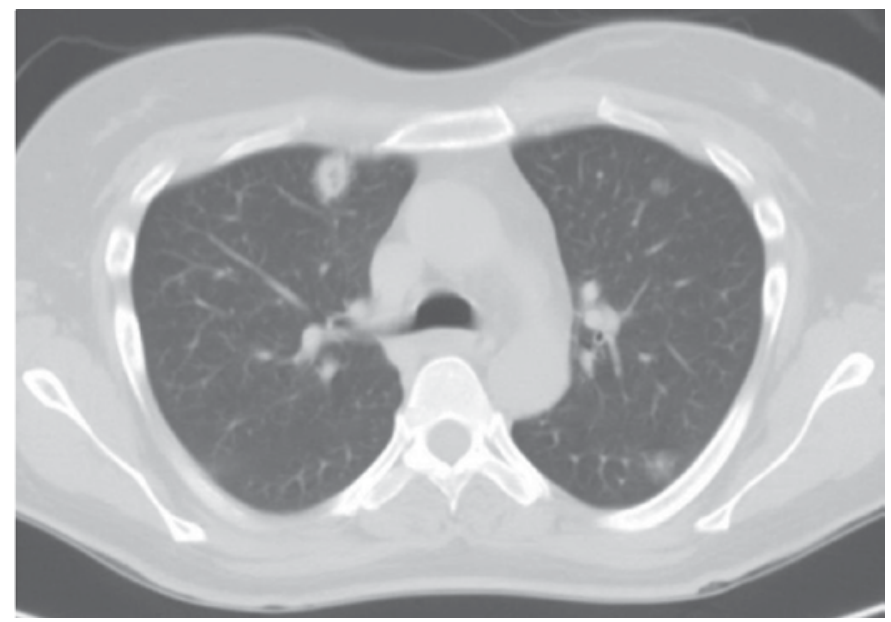

Figure 2: Computed tomography of chest showing multiple bilateral pulmonary nodules and a cavitation in the right lung.

brainstem lesions expand. On hospital day-3, a biopsy of the pulmonary lesions via video-assisted thoracoscopy (VATS) was obtained. Pathology of the pulmonary nodule revealed an angiocentric infiltrate of lymphocytes with large areas of necrosis. Within the infiltrate, certain areas showed a confluent sheet of atypical CD20-positive B cells (Figure 3). The B cells stained positive for EBV by chromogenic in situ hybridization (CISH) (Figure 4). These findings were consistent with lymphomatoid granulomatosis with areas of transformation to diffuse

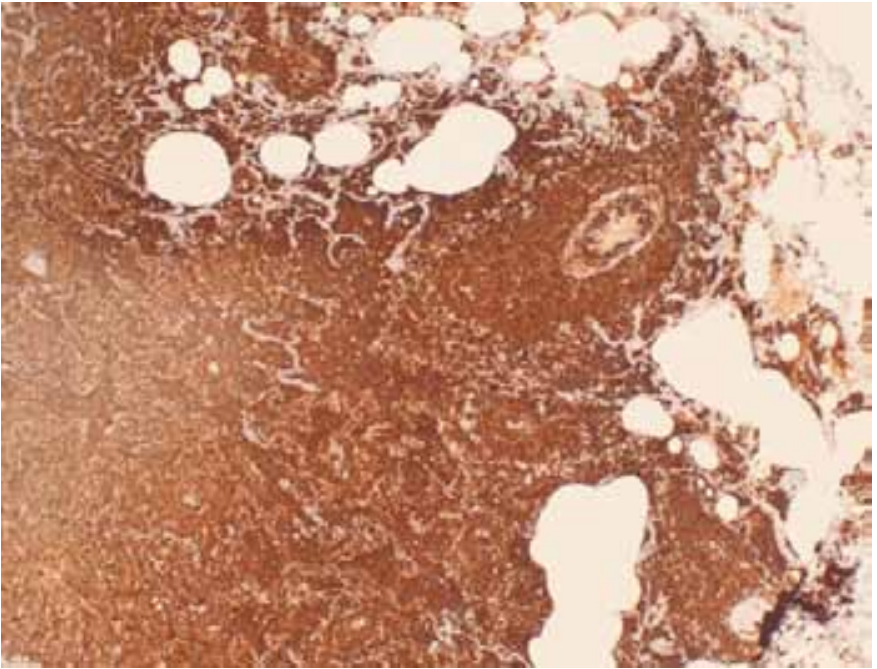

Figure 3: Area showing confluent sheet of large CD2O-positive B cells within the infiltrate (Immunohistochemistry, CD2O, x130).

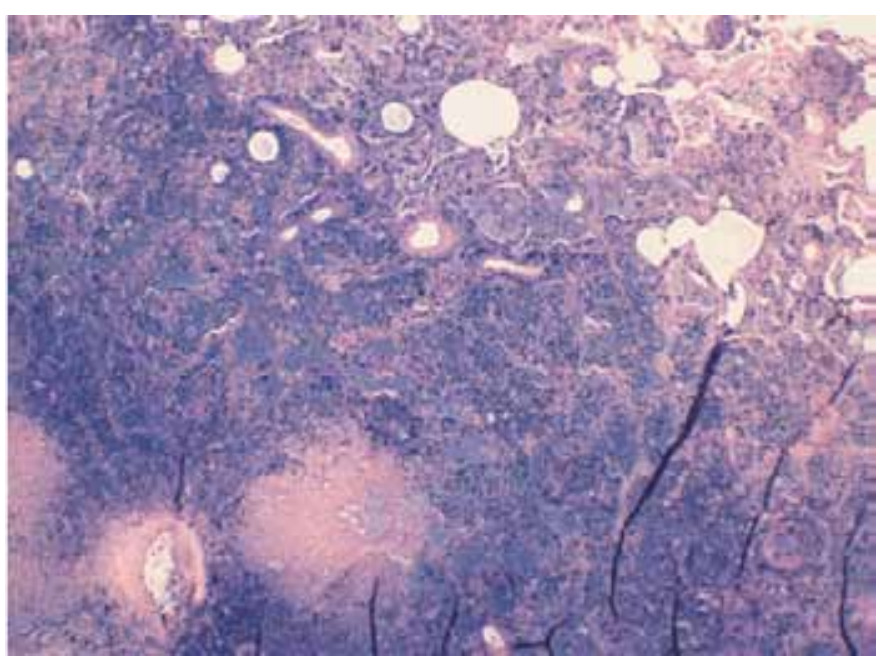

Figure 4: EBV-encoded RNA (EBER). B cells stained positive for EBV using chromogenic in situ hybridization (CISH, 130x).

large B cell lymphoma (DLBCL). Flow cytometry of the lung nodule revealed $31 \%$ aberrant $B$ cells, consistent with non-Hodgkin lymphoma. Repeated lumbar puncture on hospital day-9 revealed that CSF EBV DNA had now increased to 146,000 copies/mL and CSF flow cytometry found an aberrant population of B-lymphocytes (CD2O+, CD19+, CD10-, and lack surface immunoglobulin light chain expression) identical to those found in the lung nodule consistent with the diagnosis LYG with transformation to DLBCL. Hence a unifying diagnosis of both the pulmonary and CNS processes was made by the identical flow cytometry of the lung nodule and CSF.

\section{DISCUSSION}

Lymphomatoid granulomatosis is a rare EBVassociated extra nodal angiocentric and angiodestructive 
lymphoproliferative disorder with a high mortality rate. It was first described in 1972 by Liebow et al. as a condition that predominantly affects the lungs, with overlapping features of granulomatosis with polyangiitis (formerly Wegener's granulomatosis) and lymphoma, not fitting with other forms of lymphoma [6, 7]. LYG is classified by the 2008 WHO classification as among the diffuse large B cell lymphomas [8].

The largest case series reported that most patients with LYG are middle-aged adults with a mean age of 48 years, and the male-to-female ratio is 2:1 [5]. LYG is more common in immunodeficiency states, especially AIDS but can be seen in immunocompetent individuals as well [5]. While most HIV-infected patients who present with LYG are not on HIV therapy, it can also present as an IRIS phenomenon shortly after initiation of antiretroviral therapy [9].

Symptoms are usually present for a few months prior to diagnosis [10]. Pulmonary involvement is almost always present [11]. Chronic cough, fever and dyspnea are the most common presenting complaints. A crucial factor contributing to the frequent delay in diagnosis of LYG is its ability to mimic various infectious diseases, vasculitides, and malignancies $[5,10]$. Bilateral pulmonary infiltrates or nodules are usually seen. The nodules often have irregular margins and may cavitate, or wax and wane. Despite its lymphoproliferative nature, lymphatic tissue sites are not involved [10]. Following skin (39\%), CNS (30\%) is the most commonly affected extra-pulmonary site [11]. CNS presentations are nonspecific and varied. A retrospective review of LYG cases at the NIH found that $52 \%$ had abnormalities on brain MRI scan [12]. The variable spectrum of findings included multiple focal intraparenchymal lesions (most common) and involvement of leptomeninges and cranial nerves (next most common). These results are compatible with our patient who presented with cranial nerve findings (auditory symptoms) consistent with her initial pontine lesion, but whose final MRI scan had progressed to show multiple intraparenchymal lesions. There are several reports of LYG confined exclusively to the CNS [13].

Since the clinical findings are nonspecific, diagnosis is based on histology. The pathological hallmark of LYG is classically described as a nodular replacement of lung parenchyma by a mixed mononuclear cell infiltrate containing CD20-positive large B lymphocytes and CD3positive small lymphocytes showing prominent vascular invasion. Supportive findings include necrosis and positive in situ hybridization for Epstein-Barr encoded RNA [5]. EBV infection can be observed in biopsies from some sites while absent from others, so that its absence does not rule out the diagnosis in a proper scenario [14]. Furthermore, the same biopsy specimen may simultaneously contain EBV positive and negative areas, encouraging surgical instead of core needle biopsies [5]. The presence of EBV and the angiocentricity help differentiate from other non-Hodgkin's lymphomas [5].
In our case the repeated CSF analysis was also helpful in the diagnosis, finding that the EBV DNA level had greatly increased and malignant cells were now present on flow cytometry.

The overall prognosis of LYG is poor, with $60 \%$ of mortality and a median survival of 4 years [10] although spontaneous remissions have been reported [11]. Treatment is not standardized. In most of the published series first-line treatment consists of corticosteroid therapy, with or without cyclophosphamide [15]. Other therapies that have been attempted and reported on with mixed results include CHOP (cyclophosphamide, doxorubicin, vincristine, prednisone), CVP (cyclophosphamide, vincristine, prednisolone), rituximab (anti-CD2O monoclonal antibody), interferon $a-2 b$ and autologous stem cell transplantation.

The patient continued to deteriorate, CNS lesions progressed, and the patient developed respiratory failure on hospital day-8. Tuberculosis therapy was discontinued. HIV therapy was started on hospital day9 with raltegravir, tenofovir and emtricitabine without improvement. The oncology consultant recommended against chemotherapy due to the patients poor performance status. The patient was unable to be weaned from the ventilator. Due to the patient's extensive disease and lack of therapeutic options, the patient was finally extubated in a palliative manner, which was according to her wishes. The patient expired immediately upon extubation, which was hospital day-24 at our institution.

\section{CONCLUSION}

This patient was found to have lymphomatoid granulomatosis, a very rare Epstein-Barr virus related malignancy. Lymphomatoid granulomatosis is a predominantly pulmonary disease, but neurological symptoms can predominate. We should be aware that HIV patients, especially those who are more immunosuppressed, could have two or more disorders explaining involvement of multiple organs, or a single entity affecting multiple anatomical locations like in this case.

\section{Acknowledgements}

We thank Dr. Vishnu Reddy from the Department of Pathology of University of Alabama at Birmingham for his valuable contribution providing the histological images included in this report.

\section{Author Contributions}

Renato Quispe - Substantial contributions to conception and design, Acquisition of data, Analysis and interpretation of data, Drafting the article, Revising it critically for important intellectual content, Final approval of the version to be published 
J Martin Rodriguez - Substantial contributions to conception and design, Acquisition of data, Revising it critically for important intellectual content, Final approval of the version to be published

David McCollum - Substantial contributions to conception and design, Acquisition of data, Revising it critically for important intellectual content, Final approval of the version to be published

\section{Guarantor}

The corresponding author is the guarantor of submission.

\section{Conflict of Interest}

Authors declare no conflict of interest.

\section{Copyright}

(C) Renato Quispe et al. 2014; This article is distributed under the terms of Creative Commons attribution 3.0 License which permits unrestricted use, distribution and reproduction in any means provided the original authors and original publisher are properly credited. (Please see www.ijcasereportsandimages.com/copyright-policy.php for more information.)

\section{LIST OF ABBREVIATIONS}

LYG: LymphomatoidGranulomatosis; EBV: Epstein-Barr virus; CMV: Cytomegalovirus; HIV: Human immunodeficiency virus; AIDS: Acquired immunodeficiency syndrome; CNS: Central Nervous System; CSF: Cerebrospinal fluid; AFB: Acid-fast bacilli; PCR: Polymerase chain reaction; DLBCL: Diffuse large B cell lymphoma; HAART: Highly active antiretroviral therapy; JC: John Cunningham virus; MRI: Magnetic Resonance Imaging.

\section{REFERENCES}

1. World Health Statistics June 2012.

2. McArthur JC, Brew BJ, Nath A. Neurological complications of HIV infection. Lancet Neurol 2005 Sep;4(9):543-55.

3. Portegies P, Solod L, Cinque P, et al. Guidelines for the diagnosis and management of neurological complications of HIV infection. Eur J Neurol 2004 May;11(5):297-304.

4. Crossley KM, Brew BJ. Neurological Complications in Controlled HIV Infection. Curr Infect Dis Rep 2013 Dec;15(6):564-8.

5. Katzenstein AL, Doxtader E, Narendra S. Lymphomatoid granulomatosis: Insights gained over 4 decades. Am J Surg Pathol 2010 Dec;34(12):e35-48.

6. Liebow AA, Carrington CR, Friedman PJ. Lymphomatoid granulomatosis. Hum Pathol 1972 Dec;3(4):457-558.

7. Katzenstein AL, Peiper SC. Detection of Epstein-Barr virus genomes in lymphomatoid granulomatosis:
Analysis of 29 cases by the polymerase chain reaction technique. Mod Pathol 1990 Jul;3(4):435-1.

8. Swerdlow SH CE, Harris NL. WHO Classification of Tumours of Haematopoietic and Lymphoid Tissues. Lyon: IARC 2008:350-1. Lyon: IARC.

9. Gonzalez-Valcarcel J, Corral I, Quereda C, et al. Primary cerebral lymphomatoid granulomatosis as an immune reconstitution inflammatory syndrome in AIDS. J Neurol 2010 Dec;257(12):2106-8.

10. Cadranel J, Wislez M, Antoine M. Primary pulmonary lymphoma. Eur Respir J 2002 Sep;2O(3):750-62.

11. Katzenstein AL, Carrington CB, Liebow AA. Lymphomatoid granulomatosis: A clinicopathologic study of 152 cases. Cancer 1979 Jan;43(1):360-73.

12. Patsalides AD, Atac G, Hedge U, et al. Lymphomatoid granulomatosis: Abnormalities of the brain at MR imaging. Radiology 2005 Oct;237(1):265-73.

13. Lucantoni C, De Bonis P, Doglietto F, et al. Primary cerebral lymphomatoid granulomatosis: Report of four cases and literature review. J Neurooncol 2009 Sep;94(2):235-42.

14. Wilson WH, Kingma DW, Raffeld M, Wittes RE, Jaffe ES. Association of lymphomatoid granulomatosis with Epstein-Barr viral infection of B lymphocytes and response tointerferon-alpha 2b. Blood 1996 Jun 1;87(11):4531-7.

15. Fauci AS, Haynes BF, Costa J, Katz P, Wolff SM. Lymphomatoid Granulomatosis. Prospective clinical and therapeutic experience over 10 years. N Engl J Med 1982 Jan 14;306(2):68-74. 


\section{ABOUT THE AUTHORS}

Article citation: Quispe R, Rodriguez JM, McCollum D. Lymphomatoid granulomatosis presenting as tinnitus in a patient with acquired immunodeficiency syndrome. International Journal of Case Reports and Images 2014;5(4):264-269

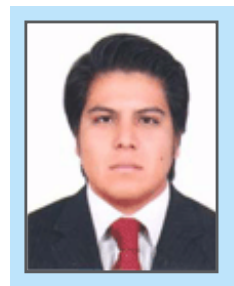

Renato Quispe is Clinical Research Associate at Instituto de Medicina Tropical "Alexander von Humboldt", Universidad Peruana Cayetano Heredia. His area of interests include tuberculosis and noncommunicable diseases. He intends to pursue an Internal Medicine residency.

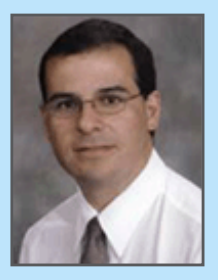

Rodriguez is Associate Professor of Medicine at the University of Alabama at Birmingham. His areas of interest are Internal Medicine and Infectious Diseases.

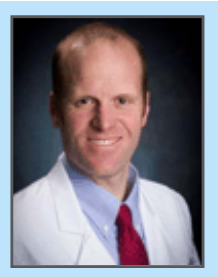

McCollum is Assistant Professor of Medicine at the University of Alabama at Birmingham. His areas of interest are Hospital Medicine and Infectious Diseases.

Access full text article on other devices

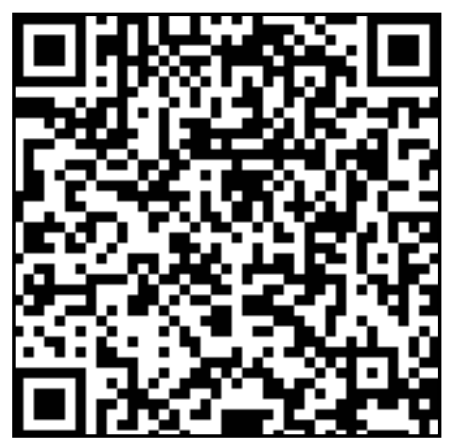

Access PDF of article on other devices

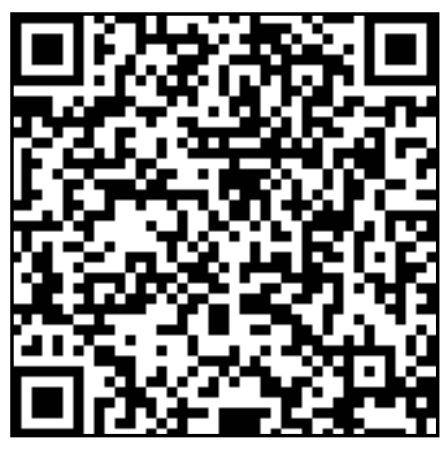

